active state, has in fact higher affinity for ribosomes than the start at the $5^{\prime}$ end of RNA 3. Two alternative explanations for the dramatic alteration in efficiency of this initiation site between RNAs 3 and 4 were discussed: either the structure of RNA 3 blocks internal initiation, or a modification at the $5^{\prime}$ end of RNA 4 is involved in ribosome binding. Isolation and sequencing of a fragment of RNA 4 which specifically binds to ribosomes led Kaesberg to opt tentatively for the second alternative: the sequence is $\mathrm{m}^{7} \mathrm{GpppGUAUUAA}$ UAAUG . . . followed by the codons for the first ten amino acids of the coat protein. A. J. Shatkin (Roche, New Jersey) pointed out in discussion that the UAAU tetranucleotide of this sequence is complementary to the common $3^{\prime}$ end of eukaryotic $18 \mathrm{~S}$ rRNA recently determined by Steitz, Shein and Delgano. Thus the mechanism of ribosome recognition may be in part similar to that used in prokaryotes. Results presented by $\mathbf{M}$. N. Thang (Institut Biologie Physico-Chimique, Paris) and by $L$. van Vloten-Doting (State Universitv Leiden). obtained with the small RNAs of the related alfalfa mosaic virus, although more preliminary, concurred with Kaesberg's conclusions on BMV.

T. Hunt (University of Cambridge) discussed the in vitro translation of tobacco mosaic virus (TMV) RNA. He and his colleagues find that the $2.2 \times$ $10^{6}$ dalton genomic RNA encodes overlapping 160,000 and 140.000 dalton proteins, but no coat protein. A small viral RNA $\left(0.25 \times 10^{6}\right)$ found in infected leaves, comprising the $3^{\prime}$ end of the total RNA, is a very efficient messenger for TMV coat protein in several in vitro systems. Among the plusstranded RNA animal viruses. two groups-I. Kennedy (University of Warwick), and L. Kääriäinen (University of Helsinki)-reported experiments with Semliki Forest virus consistent with the notion that the $42 \mathrm{~S}$ genomic RNA encodes large nonstructural proteins while the $26 \mathrm{~S}$ viral mRNA found in infected cells (comprising the $3^{\prime}$ terminal third of the $42 S$ ) is the mRNA encoding a different single polyprotein which is efficiently processed in vitro into the four viral structural proteins.

The mechanism also functions in DNA animal viruses. A. Smith (ICRF, London) and C. Prives (Weizmann Institute, Rehovot) agreed that the 19S late messenger either of SV40 or polyoma virus encodes the minor virion structural protein(s), but is an inefficient messenger for the major capsid protein (VP1). The $16 \mathrm{~S}$ late messenger, which at least in the case of polyoma virus comprises the $3^{\prime}$ terminal half of the 19S mRNA, is the messenger for VP1 synthesis in vitro. Y.Aloni recently reported at the European Tumour Virus Group meeting that the 19S SV40 mRNA is cleaved to $16 \mathrm{~S}$ in the cytoplasm, and several groups have found that it has an $m 7 \mathrm{G}$ capped $5^{\prime}$ end.

Evidence implicating $5^{\prime}$ terminal methylation in ribosome recognition was presented for VSV RNA by $\mathbf{H}$. P. Ghosh (McMaster University, Ontario) and in detail for several messengers by Shatkin. The latter reiterated his published evidence that non-methylated reovirus or VSV mRNA functions poorly in wheat germ or mouse $\mathbf{L}$ cell in vitro systems, but can be stimulated by in vitro methylation in the presence of S-adenosylmethionine in either system. He also showed that chemical removal of the terminal $m 7 \mathrm{G}$ from these viral messengers or from rabbit globin mRNA destroys messenger activity. Most interesting were direct experiments on messenger binding: the $7 \mathrm{mG}$ of reo messenger is required for binding to wheat germ ribosomes. A 35-nucleotide long fragment of reo messenger containing the $7 \mathrm{mG} 5^{\prime}$ end, which rebinds to ribosomes, was isolated from sparsomycin initiation complexes after nuclease digestion. $7 \mathrm{mG}$ has also been found in the globin mRNA ribosome binding site. Finally, Shatkin described experiments in which the $7 \mathrm{mGpppG}^{\mathrm{m}} 5^{\prime}$ terminal dinucleotide of Reo virus was extended with various mixed or homopolymers and assayed for ribosome binding. Only the

\title{
New blood parasites for old
}

from $F . E . G$. Cox

ThE malaria parasites belong to the protozoan sub-order Haemosporina which also contains a number of other blood parasites. From the earliest days of parasitology these have received considerable attention and it comes as something of a surprise to discover that until recently a whole new family has existed unrecognised in South American lizards (Lainson et al., Int. J. Parasit., 1, 241; 1971; Parasitology, 68, 117; 1974; Parasitology, 70, 119; 1975). This family is the Garniidae and its importance has grown since its discovery in 1971 partly because it looks as if it is going to contain a large number of species and partly because of the implications of the various life cycles which its members undergo. The interpretation of these life cycles throw doubts on current ideas regarding the nature of reptilian malarias and on the evolution of the malaria parasites as a whole. So far, two genera have been described, Garnia with six species and Fallisia with four.

All the other members of the Haemosporina undergo asexual division, or schizogony, in fixed cells and the malaria parasites, in addition, multiply in circulating blood cells. In the Garniidae, however, schizogony occurs only in blood cells: in red cells in Garnia and white cells in Fallisia. Some species of Fallisia occur in neutrophils and thrombocytes, cells which have not been utilised by any other parasites. Since the discovery of the exoerythrocytic stages of the malaria parasites it has been widely accepted that schizogony in the blood evolved secondarily but this belief will now have to be reassessed. In addition, all the descriptions of malaria parasites of lizards will have to be reexamined as Lainson and his colleagues have found that multiple infections with members of the Garniidae and with true pigmented malaria parasites are common and this may well have confused the original descriptions of different species. Telford (Int. J. Parasit., 3, $829 ; 1973)$ has claimed that some members of the Plasmodiidae, the malaria parasites, are not pigmented but the recent work indicates that there is no need to redefine this family in order to include non-pigmented forms.

While new blood parasites are being discovered in lizards it is likely that some widely accepted species (and even genera) in birds will have to disappear. Box (J. Protozool., 22, 165; 1975) has found that infections with the intestinal eimerian Isospora serini in canaries results in the appearance of extra-intestinal stages in blood monocytes, something that does not happen with another species $I$. canaria. Box notes the similarities between these extra-intestinal stages of Isospora and Lankesterella spp. and other eimerian parasites which occur in blood cells. This is an extension of the discovery a few years ago that Toxoplasma gondii in many mammals is an aberrant Isospora sp. of cats.

The status of all non-pigmented blood parasites will have to be looked at critically in the light of the separate studies of Box and Lainson and his colleagues and the parasites of mammals cannot be allowed to escape the scrutiny. 